\title{
Numerical simulation of cavitation flows based on their hydrodynamic similarity
}

S B Martynov*, D J Mason, and M R Heikal

School of Engineering, University of Brighton, Brighton, UK

The manuscript was accepted after revision for publication on 6 September 2005.

DOI: $10.1243 / 14680874 J E R 04105$

\begin{abstract}
Hydrodynamic similarity of cavitation flows in nozzles of different scales has been observed experimentally. In this paper a model of cavitation has been developed, taking into account the bubbly nature of cavitation and assuming local homogeneity of the vapour-liquid flow. The model of cavitation is built from correlations for evaporation and condensation, based on bubble dynamics theory, and an equation for the number density of cavitation bubbles, derived by assuming hydrodynamic similarity of cavitation flows. Compared with conventional models of cavitation, which fix the number density of cavitation bubbles, the present model takes into account the effect of liquid tension on the number density of active cavitation nuclei. The model has been implemented within the VECTIS computational fluid dynamics code and applied to the simulation of cavitating flows in nozzles.
\end{abstract}

Keywords: cavitation, modelling, similarity, scaling, liquid tension, bubbles

\section{INTRODUCTION}

In modern diesel engines, fuel injection is performed using pressures of several thousand bars through nozzles of diameter about $0.1 \mathrm{~mm}$. This is done in order to achieve high injection rates, which are necessary for superior atomization of the fuel spray and high combustion efficiency. Experimental studies [1-3] have revealed that under these conditions a decrease in pressure below a critical level in the vena contracta region [4] downstream of the nozzle entrance leads to vapour formation, which is referred to as hydrodynamic cavitation (Fig. 1). This cavitation can improve jet break-up; however, it increases the hydraulic resistance of the nozzle, produces instabilities in the flow, plus noise and vibration, and causes damage to the working surfaces (cavitation erosion) [5]. For diesel fuel injectors, identification of the parameters describing the fuel jet leaving the nozzle - velocity distribution, eddy motion, and liquid-vapour content - is essential for the accurate prediction of spray atomization. To

* Corresponding author: Engineering Research Centre, University of Brighton, Cockcroft Building, Moulsecoomb, Brighton BN2 4GJ, UK. email: s.martynov@bton.ac.uk determine these parameters at the nozzle outlet, a model for cavitation is required. To develop such a model, empirical and analytical methods have been applied [6]. Experimental studies have revealed that the behaviour of a cavitating flow strongly depends upon the particular geometry of the nozzle as well as the flow conditions. The application of numerical methods to predict this type of flow for arbitrary conditions is very attractive. However, numerical simulation of cavitation flows is still a challenging task because of the complexity of the phenomenon itself and the need for special numerical algorithms to achieve a converged solution. The present study is focused on development of the physical model and in particular the issue of hydrodynamic similarity of cavitation flows. Simulation of cavitation flow inside the nozzle is considered as a first step in the calculation of the whole injection process including the jet flow and spray break-up.

\subsection{Experimental studies of cavitation in nozzles}

For experimental data on cavitation flow to be useful in aiding the understanding of the structure of the flow, measurements of integral characteristics of the flow (such as the discharge coefficient of the nozzle) need to be combined with visualization of 

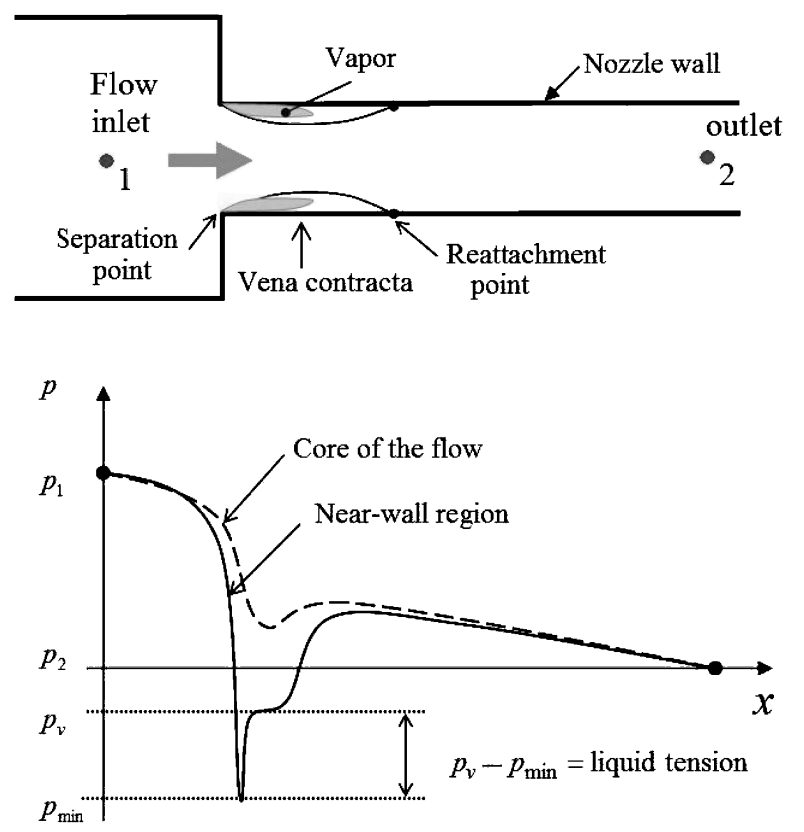

Fig. 1 Schematic representation of the geometryinduced cavitation in the nozzle and corresponding variations in pressure along the nozzle in the core of the flow and at the wall

the cavitation bubbles. Many visualization experiments have been performed using enlarged, transparent models of diesel injection nozzles [7-9]. These studies are based on the analysis of similarity of cavitation flows that allows extrapolation of the observations in the laboratory setting to naturalscale flows [10]. According to the scaling theory [5], the structure of a cavitating flow is mainly determined by the geometry of the flow (in this study, cavitation in nozzles is considered) and integral parameters, which are the cavitation number $\mathrm{CN}$ and Reynolds number, given by

$$
\begin{aligned}
& C N=\frac{p_{1}-p_{2}}{p_{2}-p_{\mathrm{v}}} \\
& \operatorname{Re}=\frac{\rho_{1} U D}{\mu_{1}}
\end{aligned}
$$

where $p_{1}$ and $p_{2}$ are the pressures upstream and downstream respectively of the nozzle (Fig. 1), $p_{\mathrm{v}}$ is the vapour pressure, usually associated with the saturation pressure in the liquid, $U=\sqrt{2\left(p_{1}-p_{2}\right) / \rho_{1}}$ is the velocity scale according to the Bernoulli equation, and $D$ is the hydraulic diameter of the nozzle.

However, experiments show that real flows do not always obey classical scaling theory. The reason for this are scale effects caused by liquid quality (the presence of dissolved gases in the liquid, small particles, and gas-vapour nuclei), bubble dynamics, geometrical differences due to manufacturing and wall roughness, the particular flow regime, and the presence of turbulent motion.

To apply the hypothesis of hydrodynamic similarity, and transfer observations from one flow to another, these scale effects have to be determined. Thus, the geometrical scale effects depend on the height of wall roughness, the shape of the inlet corners, and the degree of confinement of the flow (proximity of facing walls in the injection channel); the liquid quality effects can be described using parameters of distribution of bubble nuclei, namely their size and number density. As a result of numerous investigations, several empirical equations have been suggested to describe scale effects on the cavitation inception number (see, for example, references [1113]). At the same time, experimental studies still do not give a clear answer regarding the influence of scale effects on cavitation development. An important observation has been made by Arcoumanis et al. [9] concerning the presence of similar macroscopic two-phase structures in turbulent cavitation flows in diesel injection nozzles at natural scales and their magnified models (Fig. 2). In order to understand the nature and also quantify scale effects, the methods of computational fluid dynamics (CFD) have been applied.

\subsection{Single-fluid modelling of cavitation}

Significant progress has been achieved recently in the development of homogeneous-mixture models for the simulation of three-dimensional transient cavitating flows [14-25]. These models allow singlefluid solvers to be applied to the conservation equations for the mixture, without an increase in the computational cost compared with multi-phase simulations.

Based on a single-fluid approach, several models of cavitation, of different levels of complexity in description of the phase transition process, have been developed. Most of these imply that the local two-phase content follows the absolute pressure field. Thus, in the models based on the volume fraction transport equation $[\mathbf{1 5}, \mathbf{1 7 - 2 1}]$, the source term governing the phase transition depends on the difference between the local pressure in the mixture and the vapour pressure. The models based on the equation of state [22-25] assume an instantaneous effect of the mixture density on variations in the local pressure field. The transport-equation-based models enable the baroclinic nature of cavitation 


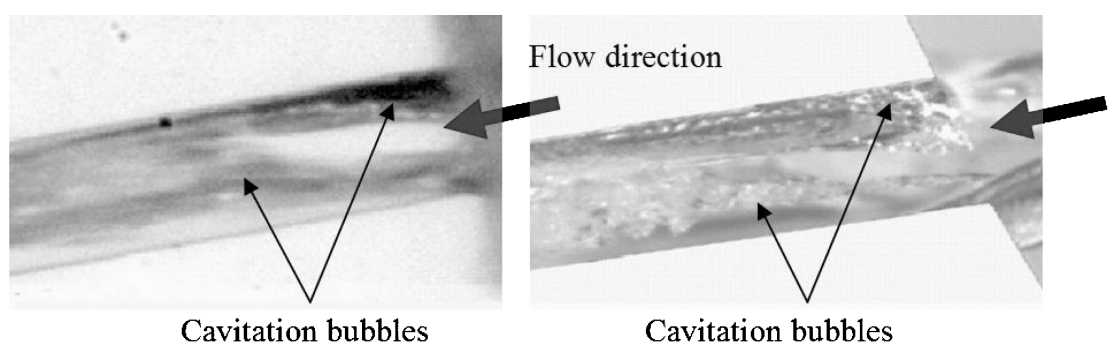

(a)

(b)

Fig. 2 Cavitation in (a) real-size diesel injection nozzle of diameter $0.176 \mathrm{~mm}$ and (b) its 20times scaled-up model $(C N \approx 5.5 ; R e \approx 12600)$ [9]

flows to be described. This type of model also allows the influence of cavitation bubble nuclei to be incorporated, which is essential for the correct description of the onset of cavitation. Transportequation-based cavitation models have been found to be robust and efficient for the prediction of cavitation flows $[\mathbf{1 7 - 2 1}]$. At the same time, these models are not as computationally expensive as non-linear models, which utilize the Rayleigh-Plesset equation of bubble dynamics theory $[\mathbf{1 4}, \mathbf{1 6}, \mathbf{2 6}]$.

Despite the above advantages of single-fluid cavitation models, comparison of a number of these [15-21,27] shows that they contain adjustable dimensional integral parameters, which are used to account for the liquid quality effects on cavitation flow. Because measurements of the liquid quality are difficult and these data are rare, description of the liquid quality effects is a problem in CFD modelling of cavitation flows.

In the models based on bubble dynamics theory, cavitation parameters characterize the spectrum of cavitation bubbles, and are commonly their initial size $R_{0}$ and volumetric number density $n[14,16,19]$. At high injection pressures, measurement of bubble nuclei of a submicron scale, and identification of the nature of cavitation nuclei (gas-vapour bubbles and the presence of particles) and the homogeneous or heterogeneous mechanism of cavitation, become difficult [16]. Distribution of cavitation nuclei in the flow depends on the pressure history, presence of impurities, and amount of dissolved gases in the liquid. The modelling of the physics concerning the bubble population dynamics complicates the model of cavitation significantly. Therefore, the conventional approach in modelling of cavitation flows $[14,16,19]$ assumes that the number density of cavitation bubbles is an integral parameter of the flow: $n=$ constant. The initial size $R_{0}$ of cavitation bubbles also is considered to be an adjustable parameter of the model. The values of these two parameters are adjusted by correlation of the results of simulations with the measurements of local and integral parameters of cavitation flows.

However, experience in simulation of different cavitation flows shows that the parameters $R_{0}$ and $n$ are not unique and can vary from one flow to another. Thus, to fit the experimental data of Roosen and Unruh [28] on cavitation in a plain, small-size nozzle, Yuan et al. [19] have applied $n=1.5 \times 10^{14} \mathrm{~m}^{-3}$ and $R_{0}=0.3 \mu \mathrm{m}$; in simulations of the flow over a hydrofoil, Basuki et al. [29] have used $n=10^{8} \mathrm{~m}^{-3}$ and $R_{0}=3 \mu \mathrm{m}$, while Frobenius et al. [30] have applied $R_{0}=30 \mu \mathrm{m}$. Following Fujimoto et al. [31], Alajbegovic et al. [32] have applied $n=10^{12} \mathrm{~m}^{-3}$ in simulations of the flow in a model of a diesel injector. The large dispersion in the values of $R_{0}$ and $n$ decreases reliability of the cavitation model and limits the range of its possible application. Although these parameters may vary from one fluid to another, the problem with models of cavitation such as those in references [16] and [19] is that hydrodynamic and liquid quality effects are merged; i.e. only one parameter, $n$, is available to tune the model for changes in either effect. Lecoffre and Bonnin [10] have shown that the liquid quality effects can be described if the number density $n$ of cavitation nuclei in the liquid is scaled by the size of the model $\ell_{\text {hydr }}$ according to

$$
n \propto \ell_{\text {hydr }}^{-3}
$$

Also, Chen and Heister [16] have reasonably concluded that, when $n$ is considered as constant for a given fluid, the non-dimensional formulation of the model contains the cavitation parameter as

$$
\tilde{n}=n \ell_{\text {hydr }}^{3}
$$

which depends on the hydrodynamic length scale $\ell_{\text {hydr }}$ of the flow. As a result, the mathematical descriptions are not identical for cavitation flows of different scales. The hydrodynamic scale $\ell_{\text {hydr }}$ is a 
typical length scale of the flow, applied in nondimensional formulations. For example, it could be the diameter of the nozzle throat or the length of the chord of a hydrofoil. It can be varied from one geometry to another and thus cannot explicitly be used in the model for the number density $n$. Correlation (3) can be considered only as a statement of the fact that the number density $n$ depends on the hydrodynamics of the flow. Development of a model for the number density $n$ that could provide identical $\tilde{n}$ for similar cavitation flows of different scales $\ell_{\text {hydr }}$ is the subject of the present study.

To achieve the hydrodynamic scaling effect, the number density of cavitation bubbles can be considered as a function of the flow conditions. In the general case, the number density of cavitation bubbles may be treated as a local flow variable, governed by a transport equation, similar to the Boltzmann equation in the theory of molecular dynamics. This will achieve the effects of formation and destruction of bubbles, their coalescence, breakup, and convection in the flow. Because of the high computational cost, this approach is developed only for one-dimensional models of cavitating, flashing, and boiling flows [33-35]. At the same time, this approach would be able to describe deviation from scaling laws for cavitation flows; however, this subject is beyond the scope of the present study.

An alternative method to describe the effect of hydrodynamic scaling (3) is to apply an algebraic equation for the number density $n$ of cavitation bubbles. In the present study an algebraic model for $n$ is developed assuming that the relationship (3) can be satisfied implicitly when both the number density $n$ and the hydrodynamic length scale are correlated with the pressure in cavitation flow.

\section{MODEL OF CAVITATING FLOW}

\subsection{Model for liquid-vapour flow}

In this study it has been assumed that the liquidvapour mixture can be described in terms of two interpenetrating continua. Thus, the flow may be simulated as either a 'multi-phase' (separate velocity vectors for the liquid and vapour) or a 'singlephase' (one velocity vector) flow. The following simplifying assumptions have been made: there is no slip between the continua and the continua are in thermal equilibrium. Thus the mixture can be considered as a 'single phase' with its physical properties varying according to the local concentration of liquid and vapour. The set of governing equations, which describes the flow of the mixture in Cartesian coordinates $[\mathbf{1 9}]$ is

$$
\begin{aligned}
& \frac{\partial \rho}{\partial t}+\frac{\partial \rho u_{j}}{\partial x_{j}}=0 \\
& \frac{\partial \rho u_{i}}{\partial t}+\frac{\partial \rho u_{i} u_{j}}{\partial x_{j}}=-\frac{\partial p}{\partial x_{i}}+\frac{\partial \tau_{i j}}{\partial x_{j}} \\
& \tau_{i j}=\left(\mu+\mu_{\mathrm{T}}\right)\left(\frac{\partial u_{i}}{\partial x_{j}}+\frac{\partial u_{j}}{\partial x_{i}}-\frac{2}{3} \delta_{i j} \frac{\partial u_{k}}{\partial x_{k}}\right)
\end{aligned}
$$

The flow is considered to be incompressible, with the properties of the liquid and vapour being constant. The mixture properties [19] are taken to be

$$
\begin{aligned}
& \rho=\alpha \rho_{\mathrm{v}}+(1-\alpha) \rho_{1} \\
& \mu=\alpha \mu_{\mathrm{v}}+(1-\alpha) \mu_{1}
\end{aligned}
$$

where $\alpha$ is the volume fraction of the vapour.

Equations (4) and (5) should be completed by the initial and boundary conditions, and the equations for calculation of the turbulent viscosity $\mu_{\mathrm{T}}$ of the mixture and the void fraction $\alpha$. The development of the transport equation for the void fraction, and source term in this equation to describe the rate of growth and decay of the vapour phase, is presented in section 2.2.

\subsection{Model for cavitation}

The present study is based on a single-fluid cavitation model [19], which has been developed under several assumptions about the nature of the cavitation phenomenon. First, the flow is described assuming a local homogeneous mixture of the vapour and liquid phases. This concept neglects the actual shape of the cavitation pockets and uses the void fraction to quantify the local content of vapour in the flow. Second, to calculate the void fraction, a transport equation is introduced [19]. In this equation the rates of evaporation and condensation are derived using an analogy with the growth and decay of spherical bubbles in liquid. To make the homogeneous concept valid, these bubbles should be small, so that their motion relative to the liquid can be neglected.

\subsubsection{Void fraction}

The current approach assumes that cavitation can be described locally for bubbles of one sort, characterized by size and number density per volume of 
liquid (monodisperse model). The volume fraction of the vapour can then be computed from the number density and radius of these virtual bubbles according to

$$
\alpha=\frac{n \frac{4}{3} \pi R^{3}}{1+n \frac{4}{3} \pi R^{3}}
$$

\subsubsection{Bubble radius}

To estimate the rates of growth and collapse of bubbles, the linear model [36] is applied and is given by

$$
\frac{\mathrm{d} R}{\mathrm{~d} t}=\sqrt{\frac{2}{3} \frac{\left|p_{\mathrm{v}}-p_{1}\right|}{\rho_{1}}} \operatorname{sgn}\left(p_{\mathrm{v}}-p_{1}\right)
$$

where $p_{\mathrm{v}}$ is the vapour pressure associated with the pressure inside the bubble and $p_{1}$ is the pressure in the surrounding liquid, approximately equal to the pressure in the mixture: $p_{1} \approx p$. This model can be considered as a limiting case of the Rayleigh-Plesset equation, when the effects of surface tension, liquid viscosity, and inertia of the bubble are neglected.

To initiate cavitation, bubble nuclei of radius $R_{0}$ are assumed to be present in the liquid.

\subsubsection{Concentration of bubbles}

According to the original model suggested by Yuan et al. [19], the number density of bubbles in equation (8) is considered as a fixed parameter for the whole flow. It is specified per volume of the liquid to account approximately for the effects of coalescence and break-up of bubbles.

\subsubsection{Transport equation for the void fraction}

Following [19], the transport equation for the void fraction $\alpha$ is derived from equation (8), under the assumptions of constant densities of the vapour and the liquid and fixed number density of cavitation bubbles in the flow domain, and is

$$
\frac{\partial \alpha}{\partial t}+\frac{\partial u_{j} \alpha}{\partial x_{j}}=S_{\alpha}
$$

where the source term is given by the equation

$$
S_{\alpha}=\frac{(1-\alpha) \rho_{1}}{\rho} \frac{n}{1+n \frac{4}{3} \pi R^{3}} \frac{\mathrm{d}}{\mathrm{d} t}\left(\frac{4}{3} \pi R^{3}\right)
$$

Taking into account equations (8) and (9), the source term $S_{\alpha}$ can be rearranged in the equivalent form, which involves only the void fraction $\alpha$ and not the bubble radius $R$, according to

$$
S_{\alpha}=\frac{f(\alpha)}{\ell_{\text {cav }}} \frac{\rho_{1}}{\rho} \sqrt{\frac{\left|p_{\mathrm{v}}-p\right|}{\rho_{1}}} \operatorname{sgn}\left(p_{\mathrm{v}}-p\right)
$$

Here, $f(\alpha)=(1-\alpha)^{4 / 3} \alpha^{2 / 3}$ and $\ell_{\text {cav }}$ is the cavitation length scale, which can be expressed through the number density $n$ of the bubble nuclei in the liquid according to

$$
\ell_{\mathrm{cav}}=\frac{1}{\sqrt{6} \sqrt[3]{\frac{4}{3} \pi n}}
$$

\subsubsection{Parameters of the model}

According to the model of Yuan et al. [19], the parameters $R_{0}$ and $n$ have to be adjusted to describe the liquid quality effects on a cavitating flow. Equation (3) shows that parameter $n$ also accounts for the hydrodynamic scaling effect. In the present study, the model for the number density of cavitation bubbles is developed to achieve automatically the scaling condition (3). The monodisperse model of cavitation [19] is extended assuming that cavitation bubbles develop from the critical cavitation nuclei in the liquid. The number density of critical cavitation nuclei is allowed to vary with the liquid tension in the cavitation region. The correlation for the cumulative spectrum of cavitation nuclei (variation in the number density with the liquid tension) is derived in the next section assuming hydrodynamic similarity for cavitation flows. As a result, the model will contain parameters responsible only for the liquid quality effect and will not be affected by the hydrodynamics of the flow. They could be adjusted once for a particular fluid and then applied for simulation of cavitation flows of this fluid in other geometries.

\section{DEVELOPMENT OF THE MODEL FOR THE NUMBER DENSITY OF CAVITATION BUBBLES}

In the present study to describe the cavitation process, an assumption about the hydrodynamic similarity of cavitation flows is applied. This assumption implies that similar cavitation flows can be described by the same set of non-dimensional governing equations. Using the length hydrodynamic scale $\ell_{\text {hydr }}$ and velocity hydrodynamic scale $U$ of mean flow, the dimensionless mass- and momentum-conservation equations of the homogeneous 
cavitation flow mixture can be presented as

$$
\begin{aligned}
& \frac{\partial \tilde{\rho}}{\partial \tilde{t}}+\frac{\partial \tilde{\rho} \tilde{u}_{j}}{\partial \tilde{x}_{j}}=0 \\
& \frac{\partial \tilde{\rho} \tilde{u}_{i}}{\partial \tilde{t}}+\frac{\partial \tilde{\rho} \tilde{u}_{i} \tilde{u}_{j}}{\partial \tilde{x}_{j}} \\
& =-\frac{1}{2} \frac{\partial \tilde{\rho}}{\partial \tilde{x}_{i}}+\frac{1}{R e} \frac{\partial}{\partial \tilde{x}_{j}}\left[\tilde{\mu}\left(1+\frac{\mu_{\mathrm{T}}}{\mu}\right)\right. \\
& \left.\times\left(\frac{\partial \tilde{u}_{i}}{\partial \tilde{x}_{j}}+\frac{\partial \tilde{u}_{j}}{\partial \tilde{x}_{i}}-\frac{2}{3} \delta_{i j} \frac{\partial \tilde{u}_{k}}{\partial \tilde{x}_{k}}\right)\right]
\end{aligned}
$$

where $\tilde{u}=u / U, \tilde{p}=\left(p-p_{2}\right) /\left(p_{1}-p_{2}\right), \tilde{x}=x / \ell_{\text {hydr }}$, and $\tilde{t}=t U / \ell_{\mathrm{hydr}}$ are dimensionless velocity, pressure, coordinate, and time respectively and $R e$ is the Reynolds number (equation (2)).

The dimensionless form of the volume fraction equation (10), combined with equations (11) and (12), is

$$
\begin{aligned}
\frac{\partial \alpha}{\partial \tilde{t}} & +\frac{\partial \alpha \tilde{u}_{k}}{\partial \tilde{x}_{k}}=\tilde{S}_{\alpha} \\
\tilde{S}_{\alpha} & =S_{\alpha} \frac{\ell_{\text {hydr }}}{U} \\
& =\left[\frac{f(\alpha)}{\ell_{\text {cav }}} \frac{\rho_{1}}{\rho} \sqrt{\frac{\left|p_{\mathrm{v}}-p\right|}{\rho_{1}}} \operatorname{sgn}\left(p_{\mathrm{v}}-p\right)\right] \frac{\ell_{\mathrm{hydr}}}{U} \\
& =\frac{\ell_{\text {hydr }}}{\ell_{\mathrm{cav}}} f(\alpha) \frac{\rho_{1}}{\rho} \sqrt{\frac{\frac{1}{2}\left|p_{\mathrm{v}}-p\right|}{\frac{1}{2} \rho_{1} U^{2}}} \operatorname{sgn}\left(p_{\mathrm{v}}-p\right) \\
& =C f(\alpha) \frac{1}{\tilde{\rho}} \sqrt{\frac{1}{2} \frac{\left|p_{\mathrm{v}}-p_{2}+p_{2}-p\right|}{p_{1}-p_{2}}} \operatorname{sgn}\left(p_{\mathrm{v}}-p\right) \\
& =\frac{C}{\sqrt{2}} \frac{f(\alpha)}{\tilde{\rho}} \sqrt{\left|\frac{p_{2}-p_{\mathrm{v}}}{p_{1}-p_{2}}+\frac{p-p_{2}}{p_{1}-p_{2}}\right|} \operatorname{sgn}\left(p_{\mathrm{v}}-p\right)
\end{aligned}
$$

which may be rewritten by applying the definitions for dimensionless pressure, $\tilde{p}=\left(p-p_{2}\right) /\left(p_{1}-p_{2}\right)$, and cavitation number $C N$ (equation (1)) to give

$$
\tilde{S}_{\alpha}=\frac{C}{\sqrt{2}} \frac{f(\alpha)}{\tilde{\rho}} \sqrt{\left|\frac{1}{C N}+\tilde{p}\right|} \operatorname{sgn}\left(p_{\mathrm{v}}-p\right)
$$

Here $\tilde{\rho}=\rho / \rho_{1}, U=\sqrt{\left(p_{1}-p_{2}\right) / 2 \rho_{1}}$, and $C=\ell_{\text {hydr }} / \ell_{\text {cav }}$ is the cavitation rate constant.

The dimensionless equations (13)-(16) describe similar cavitating flows for a given type of geometry, identical initial and boundary conditions, and specified Reynolds number $R e$ (equation (2)), cavitation number $C N$ (equation (1)), and cavitation parameter $C$ as above. While the Reynolds and cavitation numbers are the similarity criteria for the flow, the cavitation parameter $C$ is based on the cavitation length scale $\ell_{\text {cav }}$ (equation (12)), which requires modelling. To satisfy the condition $C=$ constant, the length scale $\ell_{\text {cav }}$ should follow the hydrodynamic length scale of the flow $\ell_{\text {hydr }}$ according to

$$
\ell_{\text {cav }} \propto \ell_{\text {hydr }}
$$

This conclusion was reached by Lecoffre and Bonnin [10] and expressed in correlation (3). As shown above, the model for the number density of cavitation bubbles (or, in other words, cavitation length scale $\ell_{\text {cav }}$ (equation (12))) is essential for the similarity condition (3). The following analysis is performed to develop such a model.

\subsection{Cavitation length scale}

According to equation (12), the length scale $\ell_{\text {cav }}$ is related to the distance between cavitation nuclei, and is assumed to be an independent parameter of the fluid, which is not directly linked to the hydrodynamic scale of the flow. On the other hand, $\ell_{\text {cav }}$ can be affected by the local tension $p_{\mathrm{v}}-p$ in the liquid through the density of cavitation nuclei according to

$$
n=f\left(p_{\mathrm{v}}-p\right)
$$

Therefore, the conclusion can be made that both the cavitation and the hydrodynamic length scales depend on the local pressure. Since the linkage between the pressure and the hydrodynamic length scale is established, the model for $\ell_{\text {cav }}$ can be developed on the basis of equation (17).

\subsection{Hydrodynamic length scale}

The aim of this part of analysis is to find a linkage between the hydrodynamic length scale and the pressure drop in the flow. An integral hydrodynamic length scale of the flow, which determines the cavitation rate constant $C$ in equation (16), can be associated with the hydraulic diameter of the nozzle according to

$$
\ell_{\text {hydr }} \propto D
$$

Substitution of the Bernoulli velocity scale $U$ in the definition of the Reynolds number (equation (2)), and rearranging for $D$, give

$$
D=\frac{\operatorname{Re} \mu_{1}}{\sqrt{2 \rho_{1}\left(p_{1}-p_{2}\right)}}
$$


Using equations (12), (19), and (20), the similarity condition (17) takes the form

$$
n \propto\left(p_{1}-p_{2}\right)^{3 / 2}
$$

Equation (21) shows that the bubble number density is proportional to the pressure drop across the nozzle, but according to equation (18) a relationship in terms of the local liquid tension is required. Applying a simple one-dimensional analysis for separation flow in a nozzle (Fig. 1), an estimate for the pressure drop in the vena contracta region can be made. This can be applied to determine the average tension when the liquid cavitates, $p_{\mathrm{v}}-p_{\min }$, and to put the correlation (21) in the same form as equation (18).

For a cavitation-free flow in a sharp-entrance nozzle, the pressure drop in the vena contracta region (Fig. 1) can be estimated by applying onedimensional analysis when friction losses are neglected [4] according to

$$
p_{2}-p_{\min }=\chi\left(p_{1}-p_{2}\right)
$$

where

$$
\chi=\left(\frac{C_{\mathrm{c}}^{-2}-\sigma^{2}}{C_{\mathrm{c}}^{-2}-\sigma^{2}-2\left(C_{\mathrm{c}}^{-1}-1\right)}-1\right)^{-1}
$$

is the nozzle-specific constant, which is expressed through the contraction coefficient $C_{\mathrm{c}}$ and ratio of the cross-section area of the nozzle to the area $\sigma$ of the upstream plenum.

In the limit of high injection pressures, $p_{1} \gg p_{2}$ (which is typical for diesel injection conditions, when $p_{1} \approx 10^{8} \mathrm{~Pa}$ and $p_{2} \approx 10^{6} \mathrm{~Pa}$ ), the above equation gives an approximate correlation $-p_{\min } \propto \chi p_{1}$, which on the assumption that $p_{\min } \ll p_{\mathrm{v}}$ may be rewritten in the form

$$
p_{\mathrm{v}}-p_{\min } \propto p_{1}-p_{2}
$$

(At high injection pressures, when $p_{1} \gg p_{2}$, the maximum tension in the flow can be estimated as $p_{\mathrm{v}}-p_{\min } \approx-p_{1}$.) Substitution of this correlation into equation (21) gives the modelling equation

$$
n=n_{*}\left(\frac{p_{\mathrm{v}}-p_{\min }}{p_{\mathrm{v}}}\right)^{3 / 2}
$$

where $n_{*}$ is the number density of cavitation sites when $p_{\min }=0$. The value for this parameter is specified in order to match the output of the model with experimental data from a particular nozzle. Once this value has been determined, the model can be used to simulate the flow of the same fluid through different-scale nozzles.

\section{DISCUSSION OF THE MODEL}

In the present model, to evaluate the number density of active cavitation nuclei (equation (22)), the maximum value of liquid tension in the cavitation region is used. Then, the number of bubbles per volume of liquid is fixed for the cavitation region ( $n=$ constant). This approach could be useful at least for simple quasi-steady state flows when the cavitation region is localized in the flow domain.

Equation (22) takes into account the effect of local pressure on the number of cavitation bubbles in the liquid. In comparison with the models in references [16] and [19], the present model achieves the effect of hydrodynamic scaling by equation (22), and therefore contains parameters $R_{0}$ and $n_{*}$, which depend on the liquid quality only. The fact that the present model is consistent with the requirement of hydrodynamic similarity of cavitation flows can be shown by substitution of the modelling equation (22) into the source term of equation (16) to give

$$
\begin{aligned}
\tilde{S}_{\alpha}= & \frac{\operatorname{Re}}{\ell_{\mathrm{cav}}^{*}} \frac{f(\alpha)}{2 \tilde{\rho}} \frac{\mu_{1}}{\sqrt{p_{\mathrm{v}} \rho_{1}}} \sqrt{\left|\frac{1}{C N}+\tilde{p}\right|\left(-\frac{1}{C N}-\tilde{p}_{\min }\right)} \\
& \times \operatorname{sgn}\left(p_{\mathrm{v}}-p\right)
\end{aligned}
$$

Here

$$
\ell_{\mathrm{cav}}^{*}=\frac{1}{\sqrt{6} \sqrt[3]{\frac{4}{3} \pi n_{*}}}
$$

The source term (23) does not include any hydrodynamic scales of the flow, which distinguishes the present model from a number of cavitation models found in the literature [16-19]. As a result, the set of equations (13)-(16) and (22) describes similar cavitation flows of specified geometry, $R e$ and $C N$ numbers, and initial and boundary conditions.

The present study emphasizes the effect of liquid tension on the number density of cavitation nuclei, irrespective of the particular model applied to describe the cavitation bubble dynamics. For example, equation (22) could be applied together with the model for the behaviour of cavitation bubbles, based on the Rayleigh-Plesset equation [16].

\section{VALIDATION OF THE MODEL}

To validate the model (22), comparison with the experimental data is needed. The number density of nuclei is an important property of a liquid, which can be measured [37]. In real liquids, the nuclei consist of small particles, gas bubbles, and impurities 
and can be described by a distribution function. Nuclei of radius larger than the critical value can grow, while smaller bubbles stay in the liquid [38]. The difficulties in the development of a model for the number density of cavitation nuclei are associated with the liquid quality effects and identification of the nuclei, which become active in the lowpressure region. Descriptions of the effect of nuclei population on cavitation inception and scaling are problems of practical importance, which have been the subject of numerical and experimental studies [39-41]. Results of these studies can be applied in a model of cavitation, to make the number density of cavitation nuclei a flow variable.

Numerous measurements of the number density of cavitation nuclei have been performed for water flows of different qualities under different pressure conditions. As a result of generalization of experimental data, Liu and Brennen [41] suggested an equation for the number density distribution function of bubble nuclei given by

$$
\frac{\mathrm{d} n_{0}}{\mathrm{~d} R}=N \frac{\log e}{\sqrt{2 \pi} \lambda R} \exp \left\{-\frac{1}{2}\left[\frac{\log (R / \xi)}{\lambda}\right]^{2}\right\}
$$

where $N, \xi$, and $\lambda$ are parameters. Equation (24) is a rather general approximation for the nuclei distribution function and can be applied for different fluids by appropriate choice of the parameters $N, \xi$, and $\lambda$.

For a certain range of nuclear radii, the number density of nuclei can be described by the power-law equation (Fig. 3)

$$
n_{0}=\frac{A}{R^{m}}
$$

where $A$ is a dimensional parameter.

Assuming that the bubble nuclei are filled with vapour, the critical size of nuclei - which are in equilibrium with the surrounding liquid - can be determined [38] according to

$$
R_{\mathrm{cr}}=\frac{2 \sigma}{p_{\mathrm{v}}-p}
$$

where $p_{\mathrm{v}}-p$ is the tension in the liquid.

When equation (26) is substituted into the function $n_{0}(R)$, this gives the number density of active cavitation nuclei as a function of tension in the liquid (Fig. 4). Thus, for a specific liquid tension, the concentration and size of active bubble nuclei can be found.

A power-law approximation (25) provides a correlation for the active nuclei density distribution

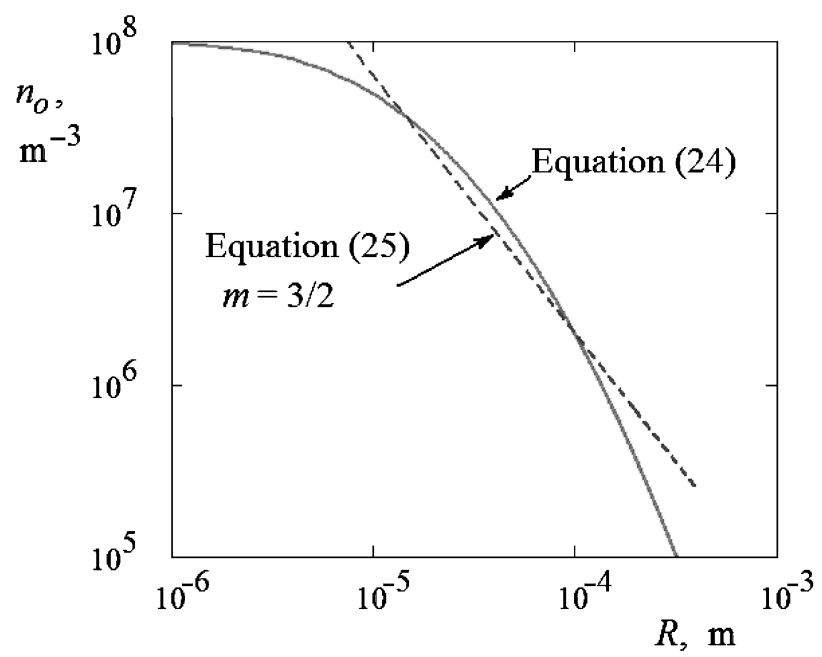

Fig. 3 Application of the power-law approximation (25) to the spectrum of nuclei described by equation (24) with the parameters $N=10^{8} \mathrm{~m}^{-3}$, $\xi=9.8 \mu \mathrm{m}$, and $\lambda=0.49[41]$

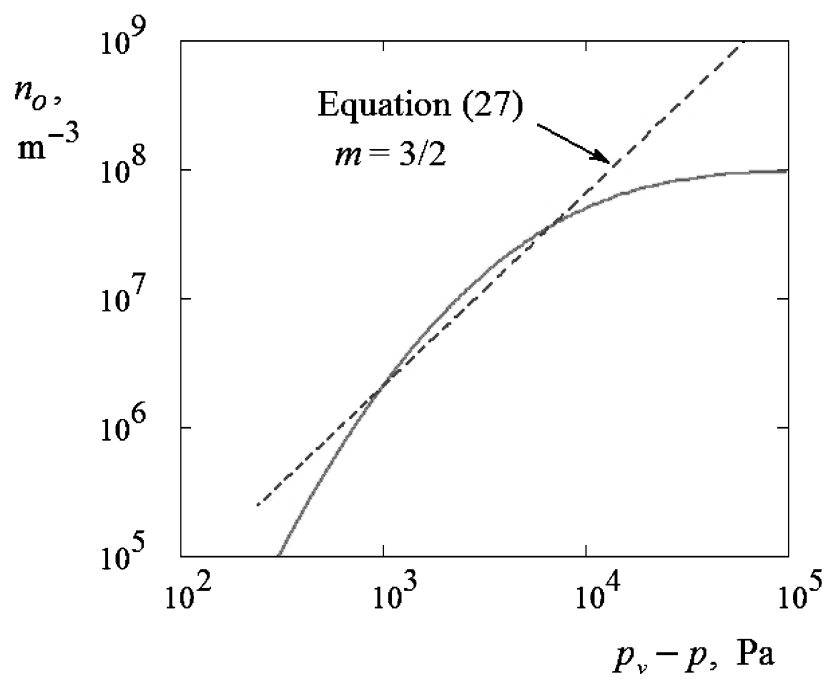

Fig. 4 Effect of local tension in a liquid on the number density of active cavitation nuclei. The solid curve corresponds to the number density of nuclei of critical size in water, described by equation (24) with the parameters specified in Fig. 3; the dashed line shows the power-law approximation (27)

function in the form

$$
n_{0}=A\left(\frac{p_{\mathrm{v}}-p}{2 \sigma}\right)^{m}
$$

Figure 4 shows that the function $n_{0}=f\left(p_{\mathrm{v}}-p\right)$ can be approximated by equation (27) with the exponent $m>0$; for bubble nuclei with radii between 0.01 and $0.1 \mathrm{~mm}$, the value $m=3 / 2$ can be applied to fit the shape of the density distribution function. More generally, Fig. 4 shows that equation (27) correctly 
describes an increase in the number density of active cavitation nuclei with the pressure drop in the liquid.

Comparison of equations (27) and (22) reveals that the latter can be considered as a result of a powerlaw approximation of an actual distribution function for bubble nuclei in the range from 0 to $R_{0}$ (Fig. 3). The parameter $n_{*}$ in the model (22) has to be adjusted to fit the density distribution function for a particular fluid. The maximum radius $R_{0}$ of cavitation nuclei in the liquid has to be specified using experimental data for cavitation inception.

\section{RESULTS OF THE MODELS VERIFICATION}

The model of cavitation (equations (4)-(12)) has been incorporated into the commercial CFD code VECTIS [42] and applied to numerical simulations of cavitation flows. Such a model could be useful to predict the amount of vapour phase, the velocity field, and the turbulence parameters of the fuel flow at the outlet of the injection nozzle.

\subsection{Description of the test}

In the following, the results of a special test designed for the modelling equation (22) are presented. In this test, the number density $n$ of cavitation bubbles is treated as the parameter of the model, which is adjusted to fit the pattern of cavitation flow, available from experiment. First, $n$ is tuned for the developing cavitation of tap water in a rectangular small-scale nozzle (Fig. 5). This step is a repetition of what was done by Yuan et al. [19]. The results of calculations are compared with the experimental data of Roosen and Unruh [28], described in reference [19]. From the results of calculations, the maximum liquid tension in the flow is applied to determine the number density parameter $n_{*}$ from equation (22). Then, the parameter $n_{*}$ is applied to predict similar cavitation flow in a 20-times-magnified nozzle of the same geometry (Table 1 ).

Numerical results have been obtained for quasisteady state cavitation flows in the nozzle shown in Fig. 5. To achieve the geometry scale effect caused by imperfections in the entrance shape of small-size nozzles, a bevelled or rounded shape of inlet corners should be specified, as was shown by Bunnel et al. [27] and Yuan et al. [19]. The shape of the nozzle entrance determines the hydrodynamics of the flow; however, it was not possible to investigate this effect thoroughly because of the absence of experimental data on the mass flowrate through the nozzle. A bevelled inlet has been specified for the nozzles. Any other scale effects on the cavitation phenomenon, concerning nucleation and bubble dynamics, roughness of the walls, etc., are not described by the model.

In this study, only cases when the vapour region does not extend throughout the nozzle were considered. This was done to avoid the need to simulate the flow inside the nozzle together with the jet flow, which is essential when modelling supercavitation [29].

Parameters of the flow used in the numerical simulations are listed in Table 1. At the inlet and outlet of the computational domain pressure, boundary conditions were set [19]. At the nozzle wall a no-slip boundary condition was used.

To describe the turbulent motion in the flow with recirculation regions, the renormalization-groupbased $k-\varepsilon$ turbulence model of Yakhot et al. [43], with wall functions, was applied as a closure to the set of governing equations. This model was chosen as one

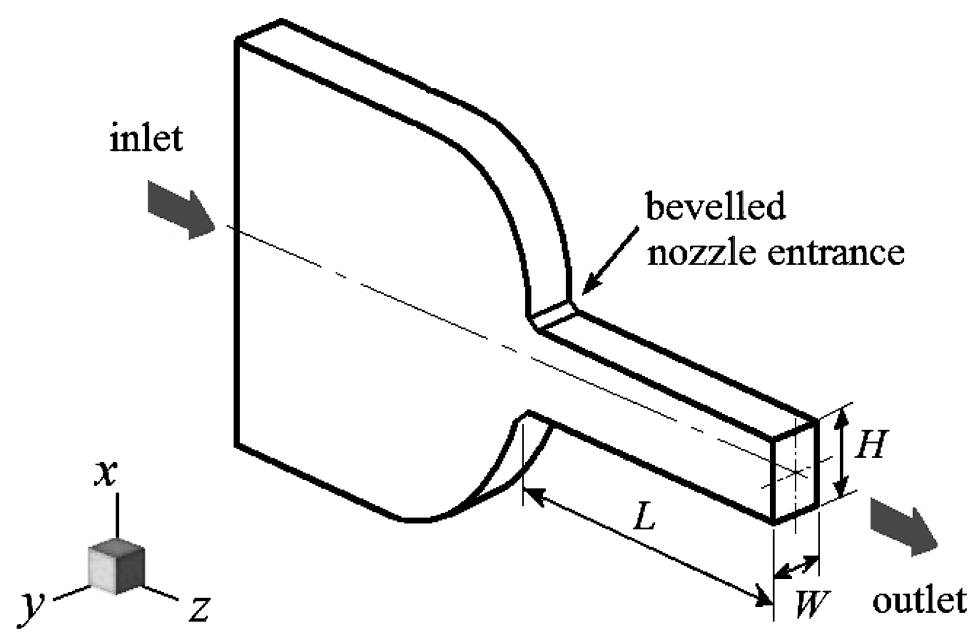

Fig. 5 Geometry and dimensions of the nozzle 
Table 1 Parameters of the cavitation flow tests

\begin{tabular}{|c|c|c|}
\hline \multirow[b]{2}{*}{ Parameter } & \multicolumn{2}{|c|}{ Value for the following } \\
\hline & $\begin{array}{l}\text { Small-scale } \\
\text { nozzle }(1: 1)\end{array}$ & $\begin{array}{l}\text { Large-scale } \\
\text { nozzle }(20: 1)\end{array}$ \\
\hline \multicolumn{3}{|l|}{ Geometrical parameters of the nozzle } \\
\hline Nozzle height $H(\mathrm{~mm})$ & 0.28 & 5.6 \\
\hline Bevelled lips height $\Delta H(\mathrm{~mm})$ & 0.01 & 0.2 \\
\hline Nozzle width $W(\mathrm{~mm})$ & 0.2 & 4 \\
\hline Nozzle length $L$ (mm) & 1 & 20 \\
\hline \multicolumn{3}{|l|}{ Parameters of the flow } \\
\hline Working liquid & \multicolumn{2}{|c|}{ Water at $20^{\circ} \mathrm{C}$} \\
\hline Inlet pressure $p_{1}(\mathrm{~Pa})$ & $80 \times 10^{5}$ & 22288 \\
\hline Outlet pressure $p_{2}(\mathrm{~Pa})$ & $21 \times 10^{5}$ & 7546 \\
\hline Bernoulli velocity scale $U=\sqrt{2\left(p_{1}-p_{2}\right) / \rho_{1}}(\mathrm{~m} / \mathrm{s})$ & 109 & 5.43 \\
\hline Saturation vapour pressure $p_{\mathrm{v}}(\mathrm{Pa})$ & \multicolumn{2}{|c|}{2300} \\
\hline$R e_{H}=\rho_{1} U H / \mu_{1}$ & \multicolumn{2}{|c|}{30350} \\
\hline$C N=\left(p_{1}-p_{2}\right) /\left(p_{2}-p_{\mathrm{v}}\right)$ & \multicolumn{2}{|c|}{2.81} \\
\hline \multirow{2}{*}{$\begin{array}{l}\text { Parameters of the cavitation model } \\
\alpha_{0}(\%) \\
\left.n \text { (nuclei } / \mathrm{m}^{3}\right)\end{array}$} & \multicolumn{2}{|c|}{0.0017} \\
\hline & $1.6 \times 10^{13}$ & $4 \times 10^{5}$ \\
\hline
\end{tabular}

of the most suitable models for simulation of flows with recirculation. Since a homogeneous mixture model is used to describe the flow, the turbulence model is applied to the mixture.

\subsection{Numerical method}

Discretization of the differential equations has been performed on a Cartesian grid with local refinement blocks. It was found that using a mesh with cells of size $\Delta x=0.0625 H$, with refinement in the region of the nozzle entry to $\Delta x=0.016 H$, was sufficient to ensure grid-independent results. The final mesh structure (one half of the nozzle in the $x-z$ plane) of approximately 163000 cells is shown in Fig. 6 .

A bounded high-order scheme [44] was used as a convection discretization scheme when solving the momentum, continuity and $k$ and $\varepsilon$ conservation equations. For discretization of the void fraction equation (11) the upwind scheme was applied [45].
In order to preserve the diagonal dominance of the matrix coefficients, the source term in the void fraction equation (11) was treated explicitly when it was negative and implicitly when it was positive [17].

To stabilize the iterations, linear under-relaxation was applied to the flow variables $\psi=u_{k}, p, k, \varepsilon$, and $\alpha$ to give

$$
\psi^{k}=\beta \psi^{k}+(1-\beta) \psi^{k-1}
$$

where $k$ is the iteration index and $\beta$ is the relaxation coefficient, which was set to 0.6 for the velocities, 0.4 for the pressure, 0.3 for $k$ and $\varepsilon$, and 0.3 for the void fraction.

As a basic pressure solver, the SIMPLER algorithm for incompressible flows was used [45]. For the vapour and liquid phases the densities were set to constants, but for the mixture the density can vary with the local void fraction, which is pressure

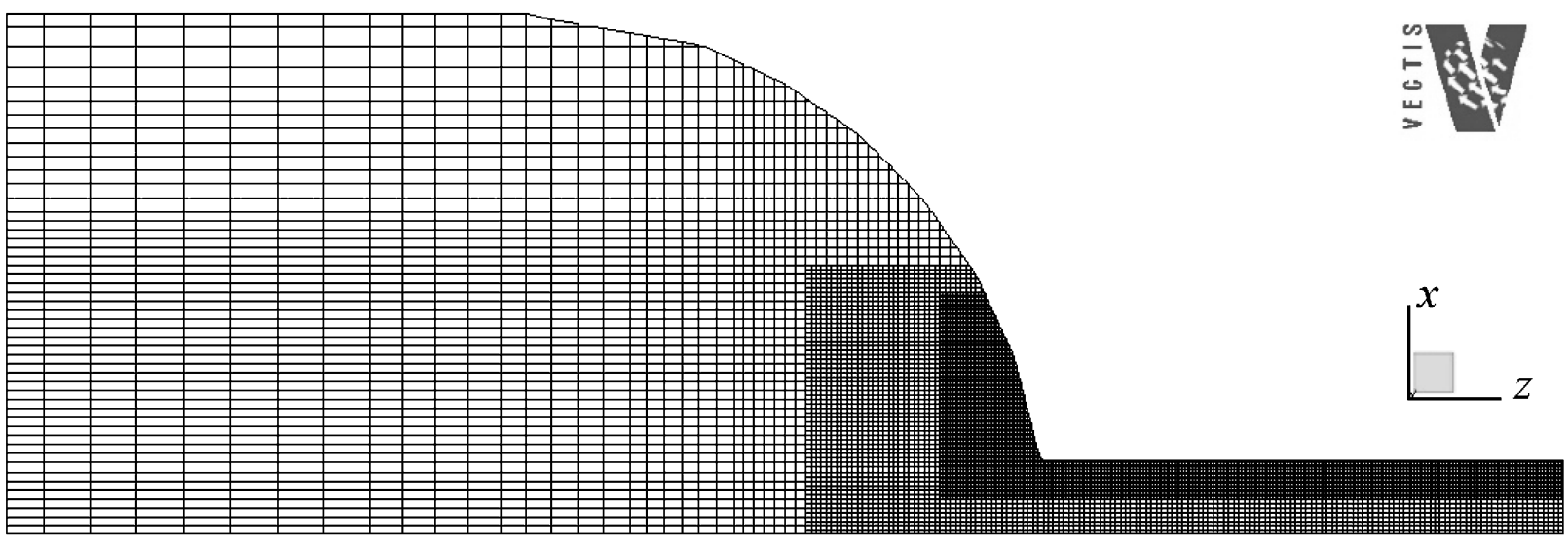

Fig. 6 The structure of Cartesian mesh in the $x-z$ plane of the computational domain (Fig. 5) 
dependent. This makes the flow of the liquidvapour mixture compressible. When using an incompressible pressure solver, large variations in density may cause large errors in the mass fluxes at cell faces, leading to imbalances in the momentum conservation and pressure correction equations. To make the computations more stable, the pressure correction equation has to be formulated in a form where density corrections are consistent with the pressure corrections. This can be achieved in two different ways. One way is to eliminate the compressibility effect completely through using an incompressible form of the continuity equation [46]. Another method accounts for the pressure-density variations directly through an additional correlation for the mixture compressibility. The mixture compressibility can be defined explicitly for the equationof-state models of cavitation $[\mathbf{2 4}, \mathbf{2 5}]$; however, it becomes a function of the flow when using the transport-equation-based models of cavitation. In this study, to couple the pressure and density variations an approximate correlation suggested by Senocak and Shyy [21] and given by

$$
\rho^{\prime}=C_{\rho} \alpha p^{\prime}
$$

is used, where $C_{\rho}$ is a numerical constant, which was adjusted to $C_{\rho}=0.1$ to obtain convergence.

\subsection{Results}

Calculations of cavitation flows were performed in two steps. First, the flow was simulated, neglecting the cavitation phenomenon. This flow field was used as the initial conditions for a simulation with the cavitation model enabled. Several iterations were needed to determine the number density $n$ of cavitation bubbles, which will match the cavitation pressure field. In general, an explicit iterative scheme can be applied to determine the parameter $n$, which would be appropriate for characterization of specific cavitation flow.

The number density $n$ is defined by equation (22), which requires an estimate of the maximum liquid tension in the cavitation region $p_{\mathrm{v}}-p_{\min }$ and knowledge of the parameter $n_{*}$. The number density parameter $n_{*}$ characterizes the liquid quality, and has to be adjusted for a specific liquid. One set of measurements of cavitation flow can be applied to determine this parameter. When it is defined, the model can be applied for other flow conditions.

In this study, the number density of cavitation bubbles $n$ was adjusted first to match the cavitation structures observed in a small-scale nozzle (Fig. 7(a)). After a number of trials, it was found that

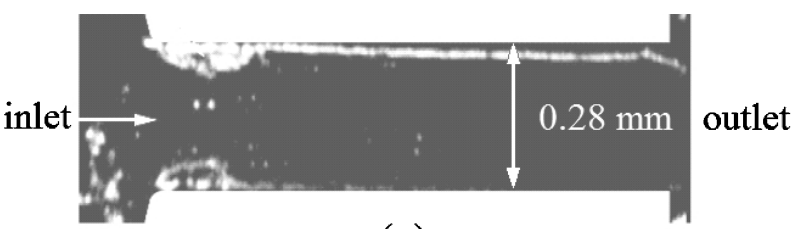

(a)

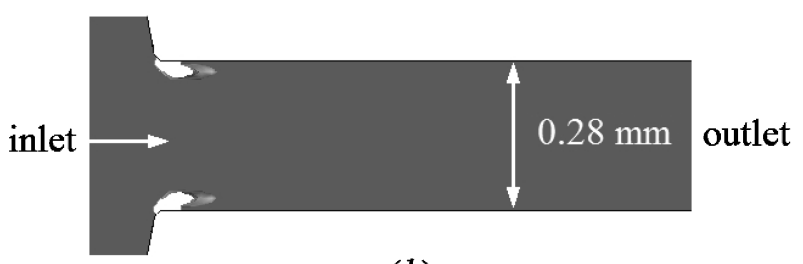

(b)

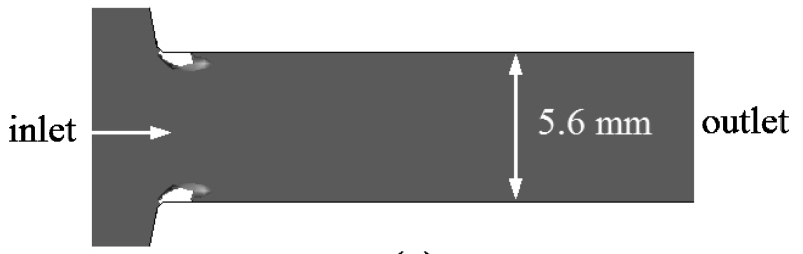

(c)

Fig. 7 Pattern of cavitating flow in (a) a small-scale nozzle (Fig. 6 of [19] (published by permission from Yuen et al.)) at $R e_{H}=\rho_{1} U H / \mu_{1}=30350$ and $C N=2.81$ in comparison with the results of numerical predictions of cavitation pockets for (b) real-scale and (c) enlarged models of the nozzle (isosurfaces; $\alpha=1$ per cent). The dark regions are liquid, and the light regions are vapour

the value $n=1.6 \times 10^{13} \mathrm{~m}^{-3}$ produced a pattern of flow in good agreement with the measured density field in the nozzle (Fig. 7). The discrepancy between the number density applied in this study and that recommended by Yuan et al. $[19]\left(n=1.5 \times 10^{14} \mathrm{~m}^{-3}\right)$ can be attributed to the difference between the inlet shapes of the nozzle and the three-dimensional flow structure of the flow considered in the present study.

The results of calculation of the maximum liquid tension $p_{\mathrm{v}}-p_{\min }$ in the cavitation region were then applied to calculate the number density parameter $n_{*}$ from equation (22), which gave $n_{*} \approx 10^{8} \mathrm{~m}^{-3}$ (Fig. 8). Knowing the number density parameter $n_{*}$, simulations were performed to predict cavitation flow in a magnified model of the nozzle (Table 1). Again, several iterations were made for the number density of cavitation bubbles in equation (22) and the degree of liquid tension $p_{\mathrm{v}}-p_{\min }$, which was not known a priori.

The results of adjustment of the number density of cavitation nuclei are presented in Fig. 9, which shows how the number density of active cavitation nuclei varies with the maximum liquid tension in the flow. 


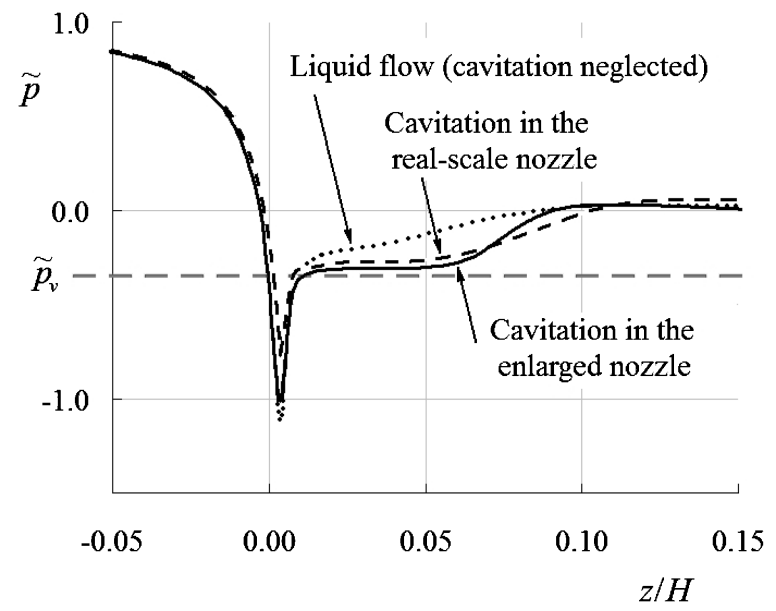

Fig. 8 Distributions of the dimensionless pressure near the wall of small- and large-scale nozzles operating under similar flow conditions (Table 1). The dotted curve shows the pressure distribution when cavitation is neglected

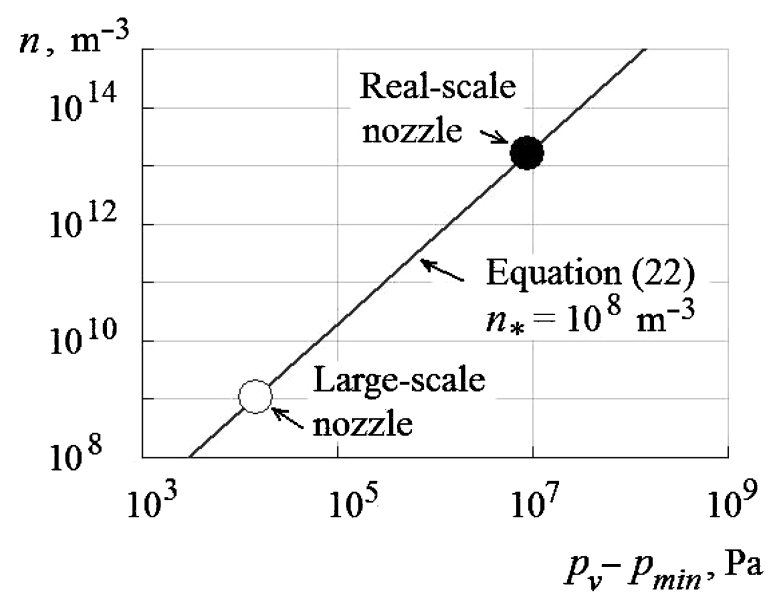

Fig. 9 Effect of liquid tension on the number density of cavitation nuclei: application of equation (22) to predict the concentration of bubbles in largescale cavitation flow

When the geometry and the flow conditions are similar to the flow, which is already known, as in the present example, there is really no need to repeat calculations for another scale. The dimensionless solution describes completely the flow field for a given set of Reynolds and cavitation numbers. However, the method described above, which adjusts the parameter $n_{*}$ first and iterates for $n$ and liquid tension second, can be recommended for prediction of cavitation flows of the same working liquid, but non-similar geometry or flow conditions.

\section{CONCLUSIONS}

To describe hydrodynamic cavitation, a homogeneous-mixture model of cavitation flow, based on the theory of bubble dynamics, has been developed. The model utilizes the differential equation for the void fraction, which contains the number density of cavitation bubbles in the liquid as a parameter.

Assuming hydrodynamic similarity of cavitation flows, an algebraic model for the number density of active cavitation nuclei (22) is suggested. The model accounts for the increase in the number of nuclei with the liquid tension, which is in agreement with experimental data [41].

The results of the numerical simulation of developing cavitation flows in nozzles of two different scales confirm that equation (22) provides a useful correlation for estimation of the number density of cavitation nuclei, which permits the hydrodynamic scaling of cavitation flows.

\section{ACKNOWLEDGEMENTS}

The authors would like to thank Ricardo UK Ltd for financial support of the project and to express their gratitude to Dr Tao Bo, who helped with the implementation of the cavitation model in the VECTIS code. Also they thank A. Shevelko for useful discussions and Professor L. A. Dombrovsky for his attention to this project and useful remarks on the text.

\section{REFERENCES}

1 Bergwerk, W. Flow pattern in diesel nozzle spray holes. Proc. Inst. Mech. Engrs, 1959, 173(25), 655660.

2 Nurick, W. H. Orifice cavitation and its effect on spray mixing. Trans. ASME, J. Fluids Engng, 1976, 98, 681-687.

3 Arai, M., Shimizu, M., and Hiroyasu, H. Breakup length and spray angle of high speed jet. In Proceedings of the Third International Conference on Liquid atomization and spray systems (ICLASS), London, 1985, pp. IB/4/1-10.

4 Chisholm, D. Two-phase flow in pipelines and heat exchangers, 1983 (Godwin, London).

5 Knapp, R. T., Daily, J. W., and Hammitt, F. G. Cavitation, 1970 (McGraw-Hill, New York).

6 Dumont, N., Simonin, O., and Habchi, C. Cavitating flow in diesel injectors and atomisation: a bibliographical review. In Proceedings of the Eighth Conference on Liquid atomization and spray systems (ICLASS 2000), Pasadena, CA, pp. 314-323. 
7 Soteriou, C., Andrews, R., and Smith, M. Direct injection diesel sprays and the effect of cavitation and hydraulic flip on atomisation. SAE paper 950080, 1995.

8 Kim, J.-H., Nishida, K., Yoshizaki, T., and Hiroyasu, H. Characterization of flows in the sac chamber and the discharge hole of a D.I. diesel injection nozzle by using a transparent model nozzle. SAE paper 972942, 1997.

9 Arcoumanis, C., Badami, M., Flora, H., and Gavaises, M. Cavitation in real-size multi-hole diesel injector nozzles. SAE paper 2000-01-1249, 2000.

10 Lecoffre, Y. and Bonnin, J. Cavitation tests and nucleation control. In Proceedings of the International Symposium on Cavitation inception, 1979, pp. 141-145 (American Society of Mechanical Engineers, New York).

11 Tullis, J. P. Cavitation scale effects for valves. J. Hydraulics Div., Proc. Am. Soc. Civ. Engrs, 1973, 99(HY7), 1109-1128.

12 Keller, A. P. Cavitation scale effects. Empirically found relations and correlation of cavitation number and hydrodynamic coefficients. In Proceedings of the Fourth International Symposium on Cavitation (CAV2001); http://cav2001.library.caltech.edu.

13 Hsiao, C.-T., Chahine, G. L., and Liu, H.-L. Scaling effect on prediction of cavitation inception in a line vortex flow. Trans. ASME, J. Fluids Engng, 2003, 125, 53-60.

14 Kubota, A., Kato, H., and Yamaguchi, H. Finitedifference analysis of unsteady cavitation on a two-dimensional hydrofoil. In Proceedings of the Fifth International Conference on Numerical ship hydrodynamics, Hiroshima, Japan, September 1990, pp. 667-684 (National Academic Press).

15 Chen, Y. and Heister, S. D. Two-phase modelling of cavitated flows. Computer Fluids, 1995, 24(7), 799-806.

16 Chen, Y. and Heister, S. D. Modelling hydrodynamic nonequilibrium in cavitating flows. Trans. ASME, J. Fluids Engng, 1996, 118, 172-178.

17 Kunz, R. F., Boger, D. A., Stinebring, D. R., Chyczewski, T. S., Lindau, J. W., Gileling, H. J., Venkateswaran, S., and Govindan, T. R. A preconditioned Navier-Stokes method for two-phase flows with application to cavitation prediction. Computer Fluids, 2000, 29, 849-875.

18 Ahuja, V., Hosangadi, A., and Arunajatesan, S. Simulations of cavitating flows using hybrid unstructured meshes. Trans. ASME, J. Fluids Engng, 2001, 123, 331-339.

19 Yuan, W., Sauer, J., and Schnerr, G. H. Modelling and computation of unsteady cavitation flows in injection nozzles. Mec. Industries, 2001, 2, 383-394.

20 Singhal, A. K., Athavale, M. M., Li, H., and Jiang Y. Mathematical basis and validation of the full cavitation model. Trans. ASME, J. Fluid Engng, 2002, 124, 617-624.

21 Senocak, I. and Shyy, W. A pressure-based method for turbulent cavitating flow computations. J. Comput. Phys., 2002, 176, 363-383.
22 Delannoy, Y. and Kueny, J. L. Two phase flow approach in unsteady cavitation modelling. In ASME cavitation and multiphase forum, 1990, FED-Vol. 98, pp. 153-158 (American Society of Mechanical Engineers, New York).

23 Avva, R. K., Singhal, A., and Gibson, D. H. An enthalpy based model of cavitation. In Cavitation and gas-liquid flow in fluid machinery devices 1995, 1995, FED-Vol. 226, pp. 63-70.

24 Schmidt, D. P., Rutland, C. J., and Corradini, M. L. A fully compressible two-dimensional model of high-speed cavitating nozzle. Atomization Sprays, 1999, 9, 255-276.

25 Dumont, N., Simonin, O., and Habchi, C. Numerical simulation of cavitating flows in diesel injectors by a homogeneous equilibrium modelling approach. In Proceedings of the Fourth International Symposium on Cavitation (CAV2001), California Institute of Technology, Pasadena, CA, 2001, pp. B6.005/1-14.

26 Delale, C. F. Thermal damping in cavitating nozzle flows. In Proceedings of the Fourth International Symposium on Cavitation (CAV2001), California Institute of Technology, Pasadena, CA, 2001, pp. B5.005/1-14.

27 Bunnel, R. A., Heister, S. D., Yen, C., and Collicot, S. H. Cavitating injector flows: validation of numerical models and simulations of pressure atomizers. Atomization Sprays, 1999, 9, 445-465.

28 Roosen, P. and Unruh, O. Cavitation-induced flow field inside fuel injector nozzles. In Proceedings of the International Symposium on Automotive technology and automation (ISATA), Düsseldorf, June 1998, pp. 267-273 (Automotive Association Ltd).

29 Basuki, W., Schnerr, G. H., and Yuan, W. Singlephase and modified turbulence models for simulation of unsteady cavitation flows. In Proceedings of the German-Japanese Workshop on Multiphase flow, Karlsruhe, Germany, 25-27 August 2002, pp. F14-F29 (Forschungszentrum, Karlsruhe $\mathrm{GmbH})$.

30 Frobenius, M., Schilling, R., Bachert, R., Stoffel, B., and Ludwig, G. Three-dimensional unsteady cavitation effects on a single hydrofoil and in a radial pump - measurements and numerical simulations. Part two - numerical simulation. In Proceedings of the Fifth International Symposium on Cavitation, 2003, paper Cav03-GS-9-005 (Kansai University, Osaka, Japan).

31 Fujimoto, H. G., Mishikori, T., Tzumakoto, T., and Senda, J. Modelling of atomization and vaporization process in flash boiling spray. In Proceedings of the Annual Conference on Liquid atomization and spray systems (ILASS-Europe 1994), paper VI-13 (Institute for Liquid Atomization and Spray Systems).

32 Alajbegovic, A., Grogger, H. A., and Philipp, H. Calculation of transient cavitation in nozzle using the two-fluid model. Proceedings of the 12th ILASSAmericas Annual Conference on Liquid atomization and spray systems, 1999, pp. 373-377 (Institute for Liquid Atomization and Spray Systems, Indianapolis). 
33 Blinkov, V. N., Jones, O. C. and Nigmatulin, B. I. Comparison with experiments using a five-equation model for vapour void development. Int. J. Multiphase Flow, 1993, 19(6), 965-986.

34 Ficarella, A. and Laforgia., D. Experimental and numerical investigation on cavitation flows in diesel injection systems. Meccanica, 1998, 33, 407-425.

35 Hibiki, T. and Ishii, M. Active nucleation site density in boiling systems. Int. J. Heat Mass Transfer, 2003, 46, 2587-2601.

36 Rayleigh, Lord. On the pressure developed in a liquid during the collapse of a spherical cavity. Phil. Mag., 1917, 34, 94-98.

37 Billet, M. L. Cavitation nuclei measurements - a review. In Cavitation and multiphase flow forum, Albuquerque, New Mexico, 1985, pp. 31-37 (ASME).

38 Brennen, C. E. Cavitation and bubble dynamics, 1995 (Oxford University Press, Oxford).

39 Rood, E. P. Review - mechanism of cavitation inception. Trans. ASME, J. Fluids Engng, 1991, 113, 163-175.

40 Gindroz, B., Bailo, G., Matera, F., and Elefante, M. Influence of the cavitation nuclei on the cavitation bucket when predicting the full-scale behavior of a marine propeller. In Proceedings of the 21st Symposium on Naval hydrodynamics, 1997, pp. 839-850 (National Academic Press, Trondheim, Norway).

41 Liu, Z. and Brennen, C. E. Cavitation nuclei population and event rates. Trans. ASME, J. Fluids Engng, 1998, 120, 728-737.

42 Ricardo Software VECTIS CFD, Version 3.8, User's manual, 2004 (Ricardo UK Ltd, Shoreham-by-Sea West Sussex).

43 Yakhot, V., Orszag, S. A., Thangham, S., Gatski, T. B., and Speziale, C. G. Development of turbulence models for shear flows by a doubleexpansion technique. Physics Fluids A, 1992, 4(7), 1510-1520

44 Zhu, J. and Rodi, W. A low dispersion and bounded convection scheme. Computer Meth. Appl. Mechanics Engng, 1991, 92, 225, 87-96.

45 Versteeg, H. K. and Malalasekera, W. An introduction to computational fluid dynamics: the finite volume method, 1995 (Longman, Harlow, Essex).

46 Spalding, D. B. A method for computing steady and unsteady flows possessing discontinuities of density. Report 910/2, CHAM, London, 1974.

\section{APPENDIX}

\section{Notation \\ C cavitation rate constant $=\ell_{\text {hydr }} / \ell_{\text {cav }}$ \\ $C N \quad$ cavitation number $\left.=\left(p_{1}-p_{2}\right) / p_{2}-p_{\mathrm{v}}\right)$}

$D \quad$ hydraulic diameter of the nozzle

$H \quad$ nozzle height

$i, j \quad$ indices of the Cartesian coordinates $=$

$1,2,3$

$\ell_{\text {cav }} \quad$ cavitation length scale

$\ell_{\text {hydr }} \quad$ hydrodynamic length scale of the flow

$L \quad$ nozzle length

$m \quad$ exponent in the power-law nuclei

distribution function (25)

$n \quad$ number density of cavitation bubbles in the the liquid $\left(\mathrm{m}^{-3}\right)$

$n_{0} \quad$ number density of cavitation nuclei in liquid $\left(\mathrm{m}^{-3}\right)$

$n_{*} \quad$ parameter in equation (22) $\left(\mathrm{m}^{-3}\right)$

$p_{1}, p_{2} \quad$ pressures upstream and downstream respectively of the nozzle

$p_{\mathrm{v}} \quad$ pressure of the vapour in the cavitation region

$p_{\min } \quad$ minimum absolute pressure in the cavitation region

$\tilde{p} \quad$ dimensionless pressure

$=\left(p-p_{2}\right) /\left(p_{1}-p_{2}\right)$

$R \quad$ radius of the cavitation bubble

Re Reynolds number $=\rho_{1} U D / \mu_{1}$

$S_{\alpha} \quad$ source term in the volume fraction equation

time

dimensionless time $=t U / \ell_{\text {hydr }}$ local velocity

dimensionless velocity $=u / U$

velocity scale according to the Bernoulli equation $=\sqrt{\left(p_{1}-p_{2}\right) /\left(\rho_{1} / 2\right)}$

$W \quad$ nozzle width

$x \quad$ coordinate

$\tilde{x} \quad$ dimensionless coordinate $=x / \ell_{\text {hydr }}$

$\delta_{i j}$

$\rho$

volume fraction of the vapour phase

Kroneker delta tensor

density

dimensionless density of the mixture

$=\rho / \rho_{1}$

$\mu \quad$ dynamic viscosity

$\tilde{\mu} \quad$ dimensionless viscosity of the mixture $=\mu / \mu_{1}$

\section{Subscripts}

$\begin{array}{ll}\text { cav } & \text { cavitation } \\ \mathrm{l} & \text { liquid } \\ \mathrm{T} & \text { turbulent } \\ \mathrm{V} & \text { vapour }\end{array}$

